\title{
Characterizing differences in students' epistemologies between classical and quantum physics.
}

\author{
Isaac E.W. Hanemann, Jessica R. Hoehn, and Noah D. Finkelstein \\ Department of Physics, University of Colorado Boulder, 390 UCB, Boulder, CO 80309
}

\begin{abstract}
In previous work, we demonstrated the existence of differences between students' epistemologies (beliefs about knowing and learning) in classical and quantum physics. Here, we explore the pervasiveness of these "splits" using five bifurcated items from the Colorado Learning Attitudes about Science Survey (CLASS) distributed across ten courses and four institutions $(\mathrm{N}=571)$. Data come from both lower and upper division quantum mechanics courses, with a variety of instructional and pedagogical contexts. Further, we investigate why students answer questions differently about classical and quantum physics through analysis of focus groups. We find splits in all course-instances surveyed. We develop a coding scheme that documents variations in epistemic beliefs between classical and quantum along themes of the following 5 categories: intuition, mathematics, social/cultural/experiential, quantum weirdness, and coherence. Lastly, we provide instances where students' epistemological splits reflect a sophisticated stance towards learning.
\end{abstract}

\section{INTRODUCTION}

Student beliefs towards physics and the nature of knowing in physics, or epistemologies, play a prominent role in student learning [1]. It has been previously shown that epistemologies vary by discipline [2]. Our prior work has found these beliefs are even finer-grained [3], where students exhibit different epistemologies, or splits, between the domains of classical and quantum physics. We confirm this sub-discipline specificity by expanding the sample of students whom we survey about classical and quantum physics. We also begin to catalogue reasons for these splits. This allows us to evaluate the impact of domain-specific epistemologies on learning physics and, ultimately, the role that instruction can play in framing these localized belief systems to benefit student learning. Furthermore, we build on prior work that has shown the sophistication or productivity of students' epistemologies are impacted by context [4]. We provide instances where the students' reasons for splitting suggest a sophisticated stance about learning quantum physics and self-awareness about where they are in the learning process. Furthermore, we argue that the presence of these splits can be valuable for the student learning process.

\section{METHODOLOGY}

Quantitative Methods. Following the methodology of Dreyfus et al. [3], we surveyed undergraduate students enrolled in modern physics and quantum mechanics on a modified CLASS [5], a measurement of student attitudes and beliefs. We bifurcated 5 items from the CLASS into two separate items, one asking about classical and one asking about quantum physics. See Table I. On the survey, all the items asking about classical physics were grouped together in a separate section section from the quantum items.

The survey was administered in ten semesters across six different courses at four universities: five semesters of lowerdivision modern physics and two semesters of upper-division quantum physics at University of Colorado Boulder (CU);
TABLE I. CLASS Items

\begin{tabular}{l|l}
\hline $\begin{array}{l}\text { CLASS } \\
\text { Item }\end{array}$ & Prompt \\
\hline \hline 6 & $\begin{array}{l}\text { Knowledge in [classical/quantum] physics consists of } \\
\text { disconnected topics. }\end{array}$ \\
\hline 23 & $\begin{array}{l}\text { In doing a [classical/quantum] physics problem, if my } \\
\text { calculation gives a very different result from what I'd } \\
\text { expect, I'd trust the calculation rather than going back } \\
\text { through the problem. }\end{array}$ \\
\hline 28 & $\begin{array}{l}\text { Learning [classical/quantum] physics changes my ideas } \\
\text { about how the world works. }\end{array}$ \\
\hline 35 & $\begin{array}{l}\text { The subject of [classical/quantum] physics has little rela- } \\
\text { tion to what I experience in the real world. }\end{array}$ \\
\hline 40 & $\begin{array}{l}\text { If I get stuck on a [classical/quantum] physics problem, } \\
\text { there is no chance I'll figure it out on my own. }\end{array}$ \\
\hline \hline CLASS &
\end{tabular}

CLASS Items included on bifurcated survey.

and one semester of upper-division quantum physics at University of Maryland (UMD), California State University Fullerton, and California Polytechnic Pomona. From this data set, two semesters of lower-division and one semester of upper-division physics at CU as well as the UMD course were included in an earlier study on split epistemologies [3]. The responses were collapsed to a 3-point Likert scale for analysis. We used the Bhapkar test [6] to determine whether the splits between classical and quantum responses for each pair of items were statistically significant.

Qualitative Methods. To begin to understand why we observe split epistemologies, we conducted one-hour focus groups for two semesters - referred to as S1 and S2 of upper-division CU physics courses surveyed (hereafter referred to as QM). QM was taught using student-centered pedagogy, including clickers and small-group tutorials. We ran the focus groups to complement quantitative findings of the existence of split epistemologies. There were 13 participants in each semester - split into two groups of four and one group of 5 - recruited from the QM class with monetary compensation, food and course extra credit. The S1 group had 4 women and 9 men, and S2 had 2 women and 11 men. 
The focus group protocol consisted of three parts: general class experience, mathematical reasoning, and the bifurcated CLASS items. For the purposes of this study, we focused on the first and third sections. The general section was comprised of several open-ended prompts, including questions about the role of math in QM and in learning quantum, the role of intuition in quantum and how they used it in QM, and about their challenges and successes. In the CLASS section, students discussed a selection of the CLASS items. For S1, we asked about items 23,35 , and 41 ; in S2, we prompted comparisons about items $6,23,28,35$, and 40. Other differences in the two focus groups include ordering of the sections, formatting of CLASS item comparison, specific experiential questions asked, and the mathematical reasoning section.

In selecting and coding responses, we define epistemological comments as any time a student made a remark on the nature and process of learning. Furthermore, these epistemological comments are identified as split if it was clear that they were speaking about quantum physics differently from classical physics. All split epistemological comments were coded with the exception of comments regarding the structure of the class (e.g. learning benefits of tutorials or a spins-first approach). Additionally, affirmations of other students' comments that did not elaborate their point are not considered epistemological comments for the purpose of our analyses; social norms can lead students to agree as a means of being supportive and polite rather than reflecting their actual feelings [7]. Using an emergent coding scheme [8], we sought to identify recurring themes that grouped the epistemological comments with minimal double-coding. Preliminary categories were presented by the lead author, reviewed collectively and revised. An independent PER researcher reviewed the final coding scheme, presented here. While these themes do not span the space of epistemological splitting nor represent preference of sub-domain, they comprise over $95 \%$ of split epistemological comments observed in the focus groups.

\section{RESULTS}

Quantitative Results. Analysis of the bifurcated CLASS survey revealed statistically significant splits $(\mathrm{p}<.05)$ for all items in at least one course-instance. Data comparison reveals that students: were less likely to see knowledge as connected in quantum, were more likely to trust the result of a calculation in quantum than work through a problem again, had their ideas about how the world works changed more by learning quantum, saw less of a relation between quantum and the real world, and felt that they were less likely to figure out a quantum problem on their own if they got stuck. Item 6 had the least number of instances with splits, splitting in 3 of the 10 post-course surveys administered, all of which were lowerdivision CU courses. Item 28 was only split in semesters taught at CU. Instances of splitting for post-instruction survey administrations are reported in Table II.

Qualitative Results. Through emergent coding, we iden-
TABLE II. Split distributions

\begin{tabular}{l|r|r|r|r|r}
\hline \hline CLASS Item & 6 & 23 & 28 & 35 & 40 \\
\hline $\begin{array}{l}\text { Course instances } \\
\text { split (out of 10) }\end{array}$ & 3 & 9 & 6 & 10 & 9 \\
\hline
\end{tabular}

Number of course-instances with statistically significant splits.

tify ten themes of epistemic splitting, grouped into five categories - intuition, math, social/cultural/experiential (SCE), quantum weirdness, and coherence. In Table III, we list the themes within each category as well as example student quotes for each theme. Names shown are pseudonyms. In total, 156 split epistemological comments were recorded. Of those, only 7 did not fit into one of the five categories. So given the questions asked in the protocol, which occupies a subset of epistemological topics that could be examined, we were able to code over $95 \%$ of comments recorded. The inclusion of percentages for categories in Table III is intended to show the comprehensiveness of the coding scheme rather than be extrapolated for a general quantum physics student population as the frequencies found in these two focus groups may not be representative of a larger population [7].

When students discussed developing a 'quantum intuition', three themes were present. Students discussed using either their classical or quantum intuition for problem-solving, suggesting that they see intuition as a domain-specific entity. Additionally, some made metacognitive comments about their growth in QM regarding their learning style, intuition, and mathematical abilities that reveal foresight and awareness of the progression of their knowledge and understanding of physics. A subset of the second theme includes students describing being wrong as more acceptable in quantum than classical. They explain that this is because they are in the process of learning quantum, whereas they feel they should know classical physics and find it more urgent to rectify knowledge gaps. The third theme is that students often describe difficulty in building a visual model for their intuition like they had in previous physics topics.

There are three themes in the math category. In addition to the visualization theme included in both intuition and math categories, students tend to trust the math more in quantum physics than classical as well as view the math in quantum mechanics as novel. Students commented on their failed attempts to visualize quantum phenomena, and how this often led to them relying on the mathematical formalism even more to shape their intuition. Another reason students discussed trusting the math more was a lack of confidence with the mathematical formalism of quantum or an intuitive expectation of outcome. Students find that they have to trust the math as they develop their intuition and move away from a classical mindset. Additionally, students say that they are less likely to do sense-making in quantum. One student states that when there's a disagreement between his calculation and his intuition, he's more likely to trust the math because "my expectation could be far from what the actual thing should be. 
TABLE III. Themes of Epistemic Splitting

\begin{tabular}{|c|c|c|}
\hline Category & Theme & Quote \\
\hline \multirow[t]{3}{*}{$\begin{array}{l}\text { Intuition } \\
81 \%\end{array}$} & $\begin{array}{l}\text { 1: Students have two distinct "intuitions", one } \\
\text { for classical and one for quantum. }\end{array}$ & $\begin{array}{l}\text { "We just learned a different intuition... The axiom of our [classical] intuition } \\
\text { comes from our experiences. But now we have a different axiom of which to } \\
\text { pull our intuition from." }\end{array}$ \\
\hline & $\begin{array}{l}\text { 2: Students recognize that they can develop in- } \\
\text { tuitions for quantum but might not be there yet. }\end{array}$ & $\begin{array}{l}\text { "It's very different to try to be intuitive about this class I think. But there are } \\
\text { some intuitions ... you can gain." }\end{array}$ \\
\hline & $\begin{array}{l}\text { 3: Students struggle and/or find new ways to } \\
\text { visualize quantum systems.* }\end{array}$ & $\begin{array}{l}\text { "We all really struggled with adjusting to not being able to visualize exactly } \\
\text { what we were doing, which was a big change from most of our other physics } \\
\text { classes." }\end{array}$ \\
\hline \multirow[t]{2}{*}{$\begin{array}{l}\text { Math } \\
73 \%\end{array}$} & $\begin{array}{l}\text { 4: Math in quantum plays a different role than } \\
\text { in classical. }\end{array}$ & $\begin{array}{l}\text { "I had a problem transitioning to the new math style cause ... it was like } \\
\text { learning a new language, especially with something that wasn't real." }\end{array}$ \\
\hline & $\begin{array}{l}\text { 5: Students tend to trust the math more in } \\
\text { quantum. }\end{array}$ & $\begin{array}{l}\text { "The math tells us that there's no hidden variables... We ... have to trust the } \\
\text { math in saying that this is actually the nature of quantum mechanics." }\end{array}$ \\
\hline \multirow{2}{*}{$\begin{array}{l}\text { Social/ } \\
\text { Culture/ } \\
\text { Experience } \\
85 \%\end{array}$} & $\begin{array}{l}\text { 6: Students describe differing levels of relation } \\
\text { to the real world. }\end{array}$ & $\begin{array}{l}\text { "Classical physics is right in my eyes. Quantum physics is kind of nonsense } \\
\text { with respect of what I see in the world." }\end{array}$ \\
\hline & $\begin{array}{l}\text { 7: Quantum has more reputation than other } \\
\text { classes }\end{array}$ & "We're kind of walking into $[\mathrm{QM}]$ expecting our brains to go on a trip." \\
\hline \multirow[t]{2}{*}{$\begin{array}{l}\text { Quantum } \\
\text { Weirdness } \\
65 \%\end{array}$} & 8: Students see quantum as weird. & $\begin{array}{l}\text { "I still am a little creeped out by that whole wavefunction collapse deal. I } \\
\text { don't really see how they ... interpreted ... that [it] just happens." }\end{array}$ \\
\hline & 9: Students see quantum as cool. & $\begin{array}{l}\text { "It's actually kind of a really beautiful theory even though everyone thinks of } \\
\text { it as this counterintuitive thing." }\end{array}$ \\
\hline $\begin{array}{l}\text { Coherence } \\
19 \%\end{array}$ & $\begin{array}{l}\text { 10: Students see differing levels of connection } \\
\text { between the topics of quantum }\end{array}$ & $\begin{array}{l}\text { "For quantum, I felt like most of it was all linked by a thread of probabilities } \\
\text { or positions... we were always looking at the same type of thing ... you're } \\
\text { always trying to measure the same things." }\end{array}$ \\
\hline
\end{tabular}

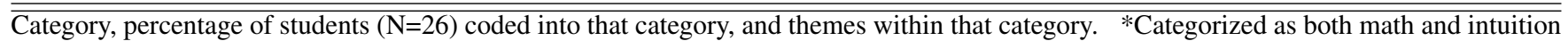

Especially in the beginning of the semester when you're not used to the quantum way of thinking, if I get a weird answer that's really different from what I expect, I'm more inclined to say ... I just don't understand this." Later in the focus group, he states "I trust the math more than my conceptual knowledge." Because they are in the process of learning the fundamentals of quantum mechanics, students often do not have intuitive expectations that they are confident in. Rather, they explain that when they get an answer that disagrees with their expectation, they identify that as an area they need to read more on. Students often noted the new mathematical representations in quantum physics were some of the more prominent distinctions between classical and quantum. Of learning Dirac notation and Hilbert space, students described QM as "a totally different game." One student remarked "I still don't know what Hilbert space is."

In the category for SCE themes, we find that students differ greatly on how they view quantum's relation to the real world. The directionality in this theme primarily arose because of students' personal interests. Most students split towards the belief that quantum has less relation to the real world than classical, citing their reasons as the intangibility of quantum effects or that quantum phenomena happen on a scale too small for them to see. Those who split towards thinking quantum had more relation generally did so because it applied to their current research. A second theme is that students comment on the reputation of quantum mechanics - particularly about the weirdness of quantum phenomena - attributable in large part to the cultural importance of quantum physics [9]. The weirdness aspect of this theme is distinct from the category on quantum weirdness because this theme describes the ideas and notions students have about quantum before taking the class. For example, students discuss having read about quantum physics before taking QM.

Comments on quantum weirdness are often related to an expectancy violation between their classical intuition and quantum results. There were stark differences in the emotion attached to the epistemic comments that students made regarding quantum weirdness, with an almost even split of students who appreciated this feature of quantum, coded as "students see quantum as cool", and those who were uncomfortable with it, coded as "students see quantum as weird." 
The final category is about the coherence of quantum. Similar to the quantum weirdness category, there were contrasting views on the coherence of quantum mechanics. To give an example of the directionality of this theme in contrast to the one provided in Table 3, we cite another student discussing that the probabilistic nature of quantum had the opposite effect. He expressed that "I think there's slightly more disconnect in [the topics of] quantum. Just cause ... you have to include probability in the physics now. And in classical, it all kinda stems from the same equations."

All 26 students involved in the focus groups had at least one response that aligned with a theme of splitting. The vast majority of students were coded for at least two categories, and most were coded for at least three. The SCE category was the most prevalent. While only a minority of students discussed a difference in the level of coherence in the topics of quantum and classical, this aligns with quantitative data that found that CLASS item 6 (knowledge is disconnected) had the fewest course-instances of post-instruction splits.

\section{DISCUSSION \& CONCLUSIONS}

The prevalence of students whose epistemologies align with the themes of epistemological splitting lends further evidence that there is often a robust distinction in student attitudes and beliefs between classical and quantum physics. The existence of splits should not be taken as inherently negative. Rather, we propose that students can still maintain epistemological sophistication when they split. They are cognizant of these splits and openly discuss that they are undergoing the process of reconciling this epistemological distinction as they gain more comfort with the material. The presence of these splits could play an important role in learning quantum. For example, many students discuss that the instructor acknowledging the weirdness of quantum allowed them to better align their expectations [9] for quantum mechanics. Also, students realizing that they cannot visualize quantum systems in the way they do for classical systems is an important step in learning quantum. This is part of deterring students from having a local-realist understanding [10] of quantum. However, students still discuss their efforts to reconcile their classical and quantum epistemologies. Some students comment that their epistemologies for quantum mechanics will more closely align with their classical epistemologies (and with experts) as they become more knowledgeable in quantum me- chanics, both conceptually and mathematically.

Many of the students who stated that they would trust the result of a calculation in quantum rather than go back through the problem would not trust the calculation in classical physics. The CLASS categorizes sense-making and effort together, but this combination may not be useful regarding split epistemologies. Some students discussed they do not go back through their calculation because they are unable to sense-make in quantum physics, not because they are unwilling to go through the effort of reconciling their expectation with the answer.

Several students in the focus group remarked that one of their favorite outcomes of taking QM is that they were able to fill the knowledge gaps that they had been left with after their introductory quantum course, such as where equations come from. Most reflected that, because they lacked the mathematical skills to understand the derivations at the time, they viewed knowledge in quantum as coming from authority. This, combined with the prominence of the belief that quantum is more mathematical than classical, suggests that a reason why we only see splits on CLASS item 6 (knowledge is disconnected) in the introductory quantum courses is that students lacked the mathematical framework to be able to piece together the concepts of quantum.

In this paper, we further validate the claim that students often have split epistemological beliefs between classical and quantum physics and provide themes that indicate reasons for splitting found through emergent coding of focus group interviews. Additionally, we have shown instances where students' reasons for splitting can be sophisticated. We highlight that students are often aware of their split beliefs and see the process of reconciling their beliefs parallel to the process of learning quantum mechanics. We consider this metacognitive reflection to be a sophisticated stance toward learning. Future work will examine the influence of instructor positionality, including pedagogical decisions such as discussion of interpretations, on the presence and reasons for splitting.

\section{ACKNOWLEDGMENTS}

The authors thank S. Pollock, J. Gifford, and the PER groups at CU and UMD for insightful discussions. We thank C. Little for his support and the students and instructors who participated in this study. Work supported by NSF STROBE STC: DMR 1548924 and NSF IUSE: 1625824.
[1] D. Hammer and A. Elby, J Learn. Sci 12, 53-90 (2003).

[2] B.K. Hofer, Int. J. Educ. Res. 45, 85-95 (2006).

[3] B.W. Dreyfus, et al., Eur. J. Phys., In Review (2018).

[4] A. Elby and D. Hammer, Epistemological resources and framing v4.1 (2010).

[5] W.K. Adams, et al., Phys. Rev. ST PER 2, 010101 (2006).
[6] X. Sun and Z. Yang, SAS Global Forum Proc., Texas (2008).

[7] N. Grudens-Schuck, et al., Extension Community and Economic Development Publications 12 (2004).

[8] V. Otero and D. Harlow, Getting Started in PER 2, 1 (2009).

[9] A. Johansson, Eur. J. Phys. 39, 025705 (2018).

[10] C. Bailey and N.D. Finkelstein, Phys. Rev. ST PER 11, 020124 (2015). 